\title{
Computational subunits in thin dendrites of pyramidal cells
}

\author{
Alon Polsky ${ }^{1}$, Bartlett W Mel $^{2} \&$ Jackie Schiller $^{1}$ \\ ${ }^{1}$ Department of Physiology, Technion Medical School, Bat-Galim, Haifa 31096, Israel. \\ ${ }^{2}$ Department of Biomedical Engineering University of Southern California
}

The thin basal and oblique dendrites of cortical pyramidal neurons receive most of the cells' synaptic input, but their integrative properties remain uncertain. Previous studies have most often reported global linear or sublinear summation. An alternative view, supported by biophysical modeling studies, holds that thin dendrites provide a layer of independent computational 'subunits' that sigmoidally modulate their inputs prior to global summation. To distinguish these possibilities, we combined confocal imaging and dual-site focal synaptic stimulation of identified thin dendrites in rat neocortical pyramidal neurons. We found that nearby inputs on the same branch summed sigmoidally, whereas widely separated inputs or inputs to different branches summed linearly. This strong spatial compartmentalization effect is incompatible with a global summation rule and provides the first experimental support for a two-layer "neural network" [The quotes are left in to refer to a standard architecture in the artificial neural network field] model of pyramidal neuron thin-branch integration. Our findings could have important implications for the computing and memory-related functions of cortical tissue. 